\title{
Tackling the different target dynamics issues in counter drone operations using passive radar
}

\author{
Tatiana Martelli, Francesca Filippini, Fabiola Colone \\ Dept. of Information Engineering, Electronics and Telecommunications (DIET) \\ Sapienza, University of Rome, Via Eudossiana 18, 00184 Rome, Italy \\ \{tatiana.martelli, francesca.filippini, fabiola.colone\}@uniroma1.it
}

\begin{abstract}
This paper deals with the detection of small unmanned air vehicles (UAVs) and drones in high-density target scenarios such as airport terminal areas. In fact, in such conditions, strong reflections by high radar cross section (RCS) targets are likely to prevent the detection of very weak target echoes. In this work, we aim at overcoming this issue by taking advantage of long coherent processing intervals (CPIs) and by implementing a CLEAN-like multistage algorithm to remove the strongest target contributions. The effectiveness of the proposed strategy is first demonstrated against simulated data representing a typical scenario. Then, a preliminary experimental result is shown, obtained against data collected by the DVB-T based AULOS $₫$ passive radar developed by Leonardo S.p.A..
\end{abstract}

Index Terms-Passive Radar, DVB-T signals, drone detection, long coherent integration time, strong targets cancellation.

\section{INTRODUCTION}

Nowadays, there is a huge interest in detecting, tracking and classifying micro-unmanned air vehicles (UAVs) and drones. In fact, their exponential use and widespread availability has redefined the security risks of critical infrastructures, national borders and military bases. Beyond their harmless uses, civilian drones have been increasingly used in problematic ways posing great threats to public security and personal privacy [1]-[2]. For example, drones can be adopted to carry explosives or other dangerous materials or to smuggle illicit materials across borders as well as for spying applications. These unauthorized low-flying objects are also becoming a serious threat for the aviation safety and the airport security and have recently caused a series of the airport shutdowns [3].

Among the different anti-drone systems, the use of Passive Radar (PR) systems can be a compelling alternative to the conventional active radars. In fact, due to the lack of an own transmitter, PR allows a number of advantages such as a reduced cost, the absence of additional electromagnetic pollution and intrinsic covert operation [4].

Among the different illuminators of opportunity (IOs) to be used, digital broadcast transmitters, such as the digital video broadcasting - terrestrial (DVB-T), are particularly attractive for drone surveillance. In particular, the high radiated power of these transmitters and the excellent coverage make them suitable for the detection of these small radar cross section (RCS) and low altitude objects. In addition, the fine range resolution of about $40 \mathrm{~m}$ make them potentially able to discriminate multiple closely spaced targets. Finally, by continuously emitting their signals, they provide a persistent illumination of the targets of interest.

The potential of a DVB-T based PR for counter drones operations has been recently investigated by different authors [5]-[9]. The reported results have demonstrated that such sensors could be effectively employed for detecting and localizing small and medium drones up to a few kilometers from the PR receiver. Moreover, the results in [8]-[9] have shown the effectiveness of the considered sensor in airport surveillance for simultaneous detection of drones flying near the airport area as well as the typical civil aircraft al farther ranges.

However, it is worth noticing that the detection of mini UAVs and drones, which are characterized by very small RCS values, might be strongly limited in high-density target scenarios. This is the case of an airport terminal area where targets of different sizes may be present.

In principle, the detection of weak target echoes might not be prevented by thermal noise since long coherent integration times could be exploited to increase the signal-to-noise ratio (SNR). Incidentally, we recall that to benefit from the use of long CPIs, proper strategies to compensate for the target migration effects must be adopted [10]. In contrast, the weak drones could be masked by stronger targets located in the same range cell or in neighbouring range-velocity cells. Note that the power received from drones can be several dBs lower than big sized targets, such as aircraft. Therefore, suitable strategies must be developed to alleviate the masking effects.

In this paper, to overcome these limitations, we take advantage of the use of long coherent processing interval (CPI) by implementing a CLEAN-like multistage algorithm that aims at removing the strongest target echoes to make the weakest ones appear and enable their detection. First, the effectiveness of the proposed strategy is investigated against a simulated case study scenario. Then, an experimental validation is carried out using the DVB-T based AULOS $®$ passive sensor developed by Leonardo S.p.A. For the purpose, a very small drone was used as cooperative target, flying in an airport terminal area. The preliminary results show that the proposed strategy effectively removes the detected strongest target echoes, thus preserving the returns from closely low RCS objects, such as drones among others.

() 2020 IEEE. Personal use of this material is permitted. Permission from IEEE must be obtained for all other uses, in any current or future media, including reprinting/republishing this material for advertising or promotional purposes, creating new collective works, for resale or redistribution to servers or lists, or reuse of any copyrighted component of this work in other works. 
The reminder of this paper is organized as follows. In Section II, we present the main motivations behind this study. Section III introduces the proposed approach and its effectiveness is shown against simulated data. Then, in Section IV, we describe the test campaign and we report the preliminary obtained results. Finally, Section V summarizes the main conclusion remarks.

\section{Motivation}

Typically, in high-density target scenarios, such as an airport terminal area, a lot of different sized objects are simultaneously present, e.g. big aircraft, small vehicles, people and potentially threatened by unauthorized drones. Therefore, the detection of low RCS targets is likely to be limited by the returns from the close and stronger ones.

As an example, we simulate a typical scenario composed by three different targets, whose echoes are received with very different SNR values as to simulate different sized objects. Note that the adopted SNR values are in line with the ones corresponding to big aircraft and small drones in DVB-T based PR, as shown from the theoretical analysis in [9]. Specifically, in the considered example, the receiver is located in the origin of the axes, the transmitter position is $\mathbf{P}_{\mathrm{TX}}=[-5 \mathrm{~km}, 0]^{\mathrm{T}}$ and each of the three point-like targets moves with constant velocity so that its position can be described by the linear law $\mathbf{P}_{\mathrm{TGT}}(\mathrm{t})=$ $\mathbf{P}_{\mathrm{TGT}}(0)+\boldsymbol{v} \cdot t, t \in[-\mathrm{T} / 2, \mathrm{~T} / 2]$, denoting $\mathrm{T}$ as the employed CPI. Details on each target are reported in Table I.

Fig. 1(a) reports an enlarged view of the bistatic rangevelocity map obtained after a conventional cross-ambiguity function $(\mathrm{CAF})$ evaluation with a $\mathrm{CPI}$ of $\mathrm{T}=0.5 \mathrm{~s}$. Observing Fig. 1(a), a strong peak with $\mathrm{SNR}=38.33 \mathrm{~dB}$ is clearly visible, which corresponds to target \#1 (see red circle in Fig. 1(a)), while a weaker return is obtained for target $\# 2$, i.e. $S N R=17.89$ $\mathrm{dB}$ (see magenta circle in Fig. 1(a)). Note that the echo from target \#2 would likely exceed the threshold for typical false alarm probability $\left(P_{f a}\right)$ values against thermal noise. However, due to its closeness to the strongest target, it is likely to be masked by its sidelobes which are comparable to it. On the other hand, since target \#3 is received with a very low SNR value, it is not even visible in the map, as highlighted by the dashed orange circle in Fig. 1(a).

Based on the considerations made on Fig. 1(a), it is clear that the use of longer CPIs would be needed in order to (i) increase the SNR of the weakest target (\#3) and (ii) increase the Doppler resolution thus enabling the discrimination of target \#2 across the velocity domain. However, it is well known that, to benefit from the use of long CPIs, the target migration effects must be properly compensated.

Table I Details of the simulated scenario

\begin{tabular}{ccc}
\hline \hline Target & $\mathbf{P}_{\mathrm{TGT}}(0)[\mathrm{m}]$ & $\boldsymbol{v}=\left[v_{x}, v_{y}\right][\mathrm{m} / \mathrm{s}]$ \\
\hline$\# 1$ & {$\left[2.6 \cdot 10^{3},-50\right]$} & {$[12,60]$} \\
$\# 2$ & {$\left[2.65 \cdot 10^{3}, 30\right]$} & {$[13,-30]$} \\
$\# 3$ & {$\left[2.6 \cdot 10^{3},-9\right]$} & {$[8,9]$} \\
\hline
\end{tabular}

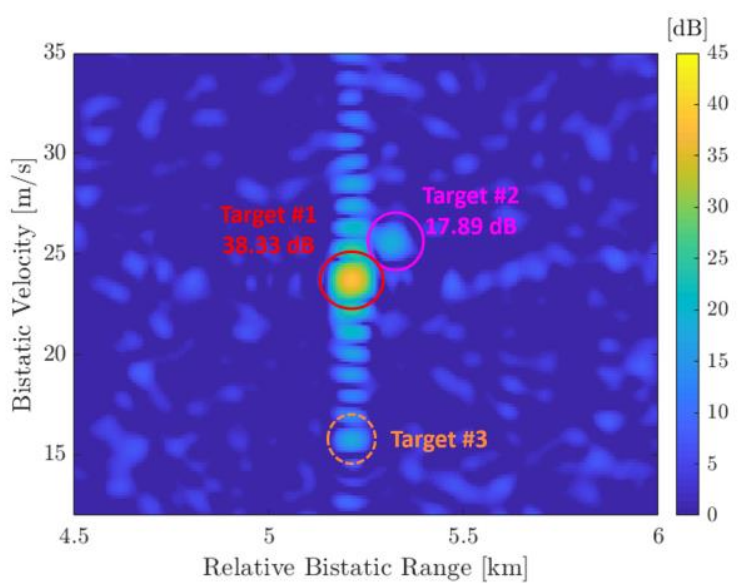

(a)

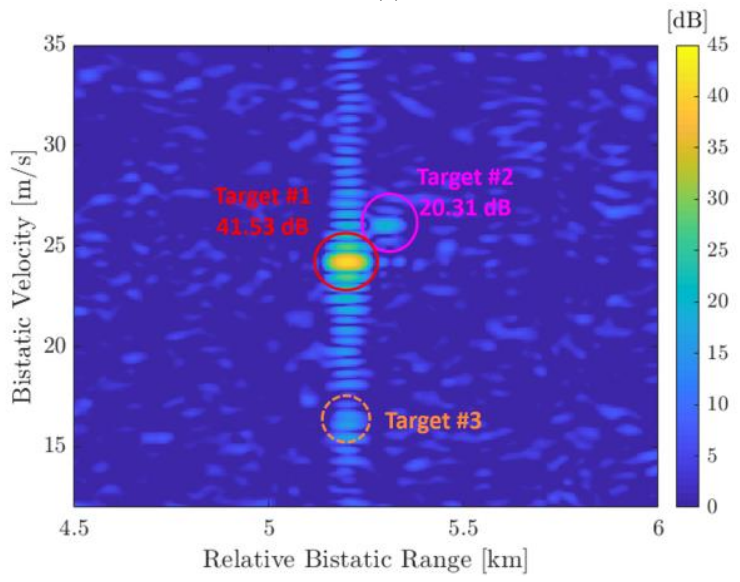

(b)

Fig. 1 Bistatic range - velocity map $\chi^{(0)}[r, v]$ obtained with

(a) $\mathrm{T}=0.5 \mathrm{~s}$ and (b) $\mathrm{T}=1 \mathrm{~s}$ after target migration compensation

To this aim, we resort to the approach described in [10] based on the compensation of the target range and Doppler migration effects. Specifically, since the target Doppler rate is unknown at the detection stage, a bank of filters is exploited for an appropriate set of Doppler rate values. In detail, we consider a grid of $\mathrm{N}_{\alpha}$ Doppler rate values $\left\{\alpha_{0}, \ldots, \alpha_{\mathrm{N}_{\alpha}-1}\right\}$, spaced by $\delta \alpha=\frac{\lambda}{T^{2}}$, where $\lambda$ is the wavelength, according to the depth of focus criterion. Then, based on the simplifying assumption that maximum one target is present at each range cell, we perform a maximization across both Doppler and Doppler rate dimensions. In other words, each range cell of the map reported in Fig. 1(b) has been focused according to the Doppler rate $\alpha^{*}$ resulting from the mentioned maximization. Additional details are reported in [10].

We show the result in Fig. 1(b) for a CPI of $T=1 \mathrm{~s}$. By observing Fig. 1(b), the following considerations are in order:

- the target \#1 motion effects have been properly compensated and the expected coherent integration gain of approx $3 \mathrm{~dB}$ is reached.

- Target \#2 benifts from both the coherent integration gain and the enhanced bistatic velocity resolution. However, its peak still is comparable to the target \#1 sidelobes in the Doppler domain.

(C) 2020 IEEE. Personal use of this material is permitted. Permission from IEEE must be obtained for all other uses, in any current or future media, including reprinting/republishing

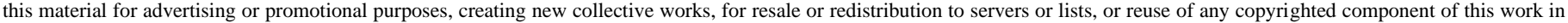
other works. 
- The increased Doppler resolution might also help target \#3, which lies on the same bistatic range cell as target \#1. However, being focused according to Doppler rate value of the strongest object, its peak is further degradated with respect to the result in Fig. 1(a) and it still buried by the sidelobes.

Based on these considerations, it is clear that the detection of the weakest targets is not only limited by thermal noise. Therefore, the only use of long CPIs is not enough if additional strategies to counteract the strongest target echoes are not considered.

We investigate in the following Section the possibility to resort to a CLEAN-like strategy to remove the strong targets contributions to enable the detection of the small RCS objects.

\section{PROPOSED SOLUTION}

This Section presents the considered signal processing strategy. First, we briefly detail the main signal processing steps that have been applied. Then, we show the results obtained on the simulated case study considered in Section II.

\section{A. Signal Processing}

The block diagram of the signal processing steps foreseen is shown in Fig. 2.

First, the signals collected by means of the reference and surveillance antennas undergo the main processing stages of a conventional PR processing, namely the Disturbance Cancellation stage and the range velocity map evaluation. We recall that the first is aimed at removing the direct signal contribution, as well as clutter and multipath [11] while the second one is aimed at evaluating the CAF between the reference and the surveillance signal. We refer to the latter as $\chi[r, v]$, with $r$ and $v$ representing the generic bistatic range velocity bin. For targets with a dominant radial velocity, the $\mathrm{CAF}$ evaluation might also include a range migration compensation (RMC) stage.

Afterwards, a CLEAN-like algorithm [12] is used with the purpose of estimating the dominant target echoes and progressively cleaning them off the image for the weak target returns to appear.

At the $i$ th iteration of the proposed algorithm, the following steps must be performed:

1. At the beginning $(i=0)$, the range-velocity map $\chi^{(0)}[r, v]$ undergoes a first thresholding stage that aims at identifying the target echoes that are stronger than a fixed power threshold $\mu$.

2. Once a strong target echo has been identified at $\left(r_{0}, v_{0}\right)$, its range cell, i.e. $\chi^{(i)}\left[r_{0}, v\right]$, is fed in iput to an inverse fast Fourier transform (IFFT) to move from Doppler frequency to the slow-time domain.

3. The output of this stage undergoes a focusing stage, using the Doppler rate value $\alpha^{*}$ that results in the maximum target peak, as described in Section II.

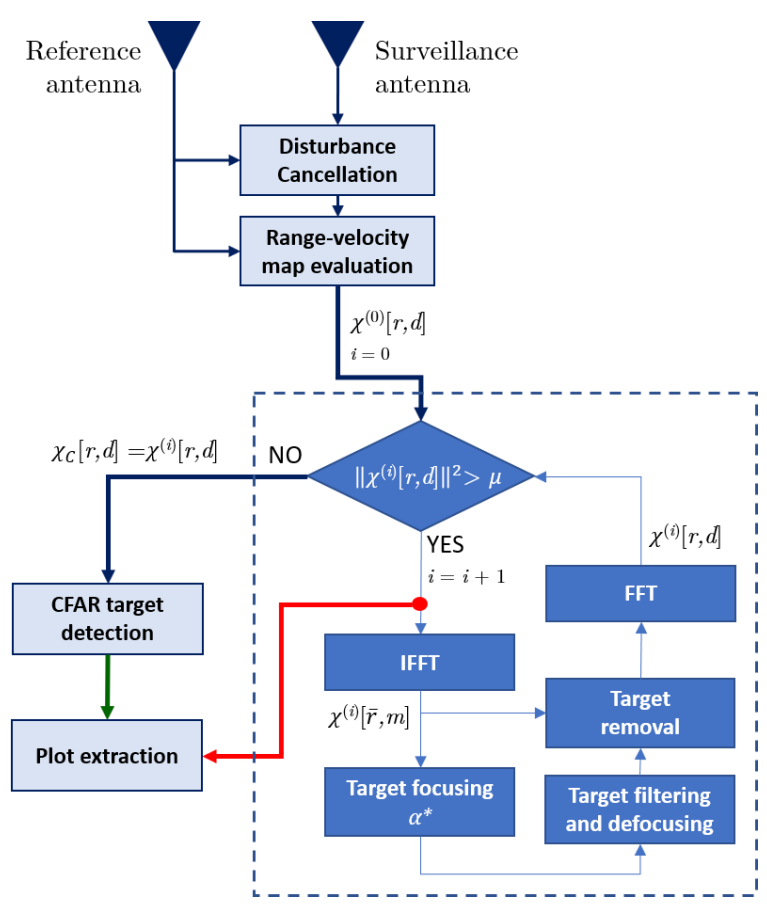

Fig. 2 Block diagram of the employed signal processing scheme

4. Then, the target contribution is properly filtered and a reverse focusing is applied to recover the current estimate of the target signal in the range-compressed slow-time domain.

5. Finally, the filtered and defocused target contribution is subtracted from the original and the new cleaned version undergoes an FFT to move from slow-time to Doppler frequency domain.

6. The obtained range-velocity map $\chi^{(i)}[r, v]$ undergoes the thresholding stage described at point 2 .

The algorithm should be arrested at the time that the bistatic range-velocity maps is likely to contain only weak target echoes namely when the threshold $\mu$ is not exceeded by any rangevelocity bin.

Once the considered algorithm has been performed, an adaptive target detection stage is applied to the cleaned bistatic range-velocity map $\chi_{C}[r, v]$, according to the approach described in Section II. Finally, the declared target detections will go in input to the plot extraction stage, along with the strongest target ones, resulting from stage 3 of the considered algorithm.

\section{B. Results}

The proposed multistage algorithm is applied against the simulated scenario described in Section II. Specifically, the fixed threshold $\mu$ has been set to $20 \mathrm{~dB}$ in order to obtain a cleaned CAF where the contribution of the target \#1 and its sidelobes have been removed. In Fig. 3 we report the Doppler cut obtained in the range bin $r_{0}$ where both target\#1 and target \#3 lie.

(c) 2020 IEEE. Personal use of this material is permitted. Permission from IEEE must be obtained for all other uses, in any current or future media, including reprinting/republishing

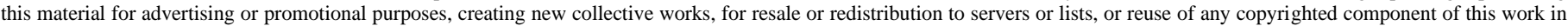
other works. 


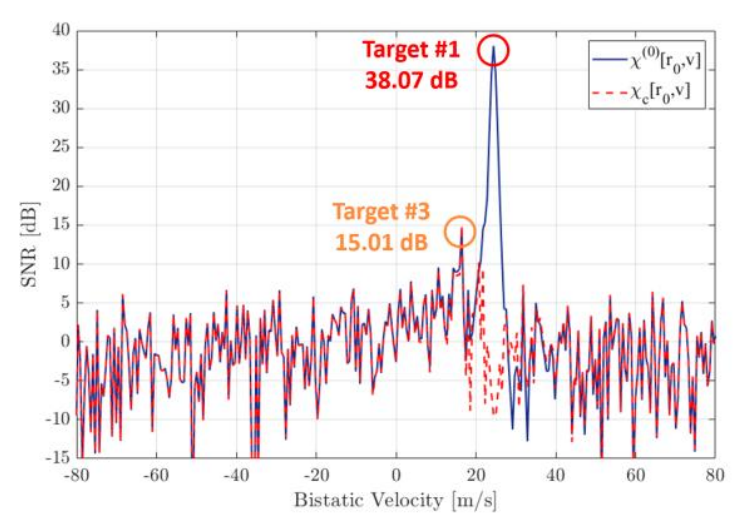

Fig. 3 Comparison between $\chi^{(0)}\left[r_{0}, v\right]$ and $\chi_{C}\left[r_{0}, v\right]$

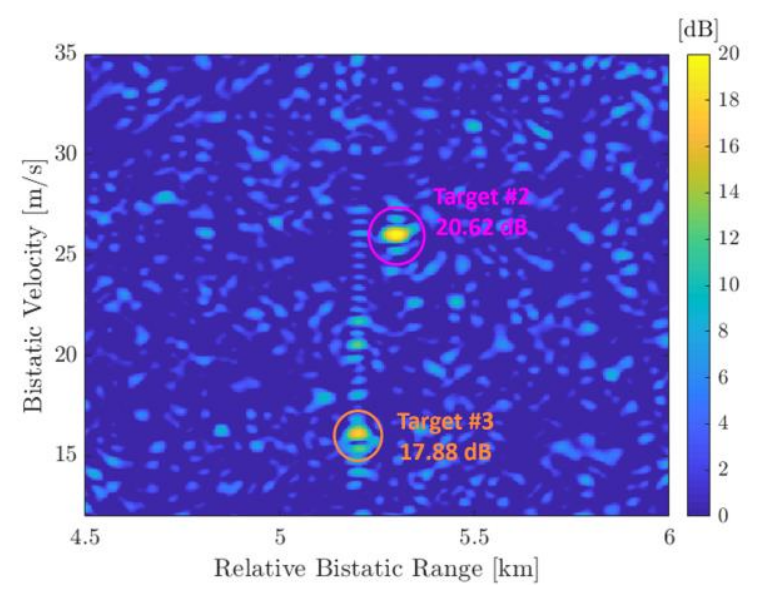

Fig. 4 Bistatic range- velocity map obtained after target migration compensation performed on $\chi_{C}[r, v]$

Specifically, we plot $\chi^{(0)}\left[r_{0}, v\right]$ in blue line, representing the input to the proposed algorithm. Note that, an RMC stage has been applied. On the same figure, we plot its cleaned version, i.e. $\chi_{C}\left[r_{0}, v\right]$, in dashed red. In this case, the algorithm is stopped after the first iteration, i.e. $\chi_{C}[r, v]=\chi^{(1)}[r, v]$, since the threshold $\mu$ is no longer exceeded by any range-velocity bin.

The following considerations apply:

(i) The proposed solution effectively removes target \#1 creating a deep notch in corrispondance to its bistatic velocity position.

(ii) Target \#3 is preserved (see orange circle) and now represents the highest peak in the considered range cell $(15.01 \mathrm{~dB})$.

(iii) Due to the motion of target \#3 which is characterized by a non-negligible tangential velocity (see Table I), further improvements are expected if the Doppler walk effect is also properly corrected.

Therefore, following the block diagram sketched in Fig. 2, the cleaned map $\chi_{C}[r, v]$ undergoes the detection stage, which includes the Doppler migration compensation (DMC) [10]. Fig. 4 shows the result range-velocity map in input to the adaptive

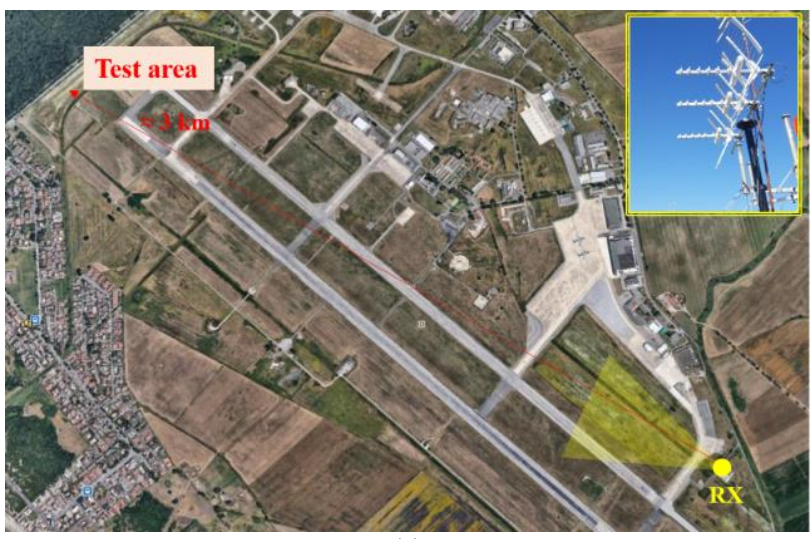

(a)

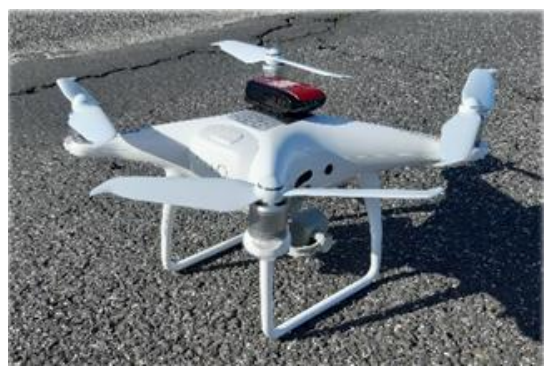

(b)

Fig. 5 Experimental scenario: (a) Sketch of the acquisition geometry (b) DJI Phantom 4 Pro used as cooperative target

thresholding stage, namely after the Doppler walk effect has been compensated. After this stage, both target \#2 and target \#3 are clearly visible as the highest peaks in the map and are likely to exceed the adaptive threshold for typical $P_{f a}$ values.

\section{EXPERIMENTAL VALIDATION}

In this Section, preliminary experimental results are presented. First, the acquisition campaign is briefly described, then we show some experimental results.

\section{A. Acquisition campaign}

The dataset considered in this work has been collected during an acquisition campaign carried out at the military airport of Pratica di Mare, Italy, using the DVB-T based AULOS ${ }^{\circledR}$ PR system, developed by Leonardo S.p.A.. The DVB-T transmitter of Monte Cavo (about $22 \mathrm{~km}$ away) has been employed as IO while the sensor was installed very close to the runway of the airport. Fig. 5(a) reports a sketch of the acquisition geometry. During the test campaigns, three Yagi-Uda receiving antennas have been employed as surveillance antennas, each one characterized by a main beam of $36^{\circ}$.

The considered dataset is composed by 87 consecutive data files, each of 2.3s. During the acquisition time, a small drone, namely a DJI Phantom 4 Pro of size approx. $25 \mathrm{~cm}$ x $25 \mathrm{~cm}$ was used as cooperative target (see Fig. 5(b)). It was equipped with a GPS receiver to record its position. As illustrated in Fig. 5(a), the drone flew at approx. $3 \mathrm{~km}$ away from the PR sensor.

(C) 2020 IEEE. Personal use of this material is permitted. Permission from IEEE must be obtained for all other uses, in any current or future media, including reprinting/republishing

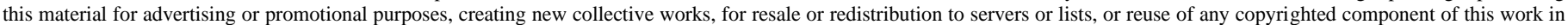
other works. 


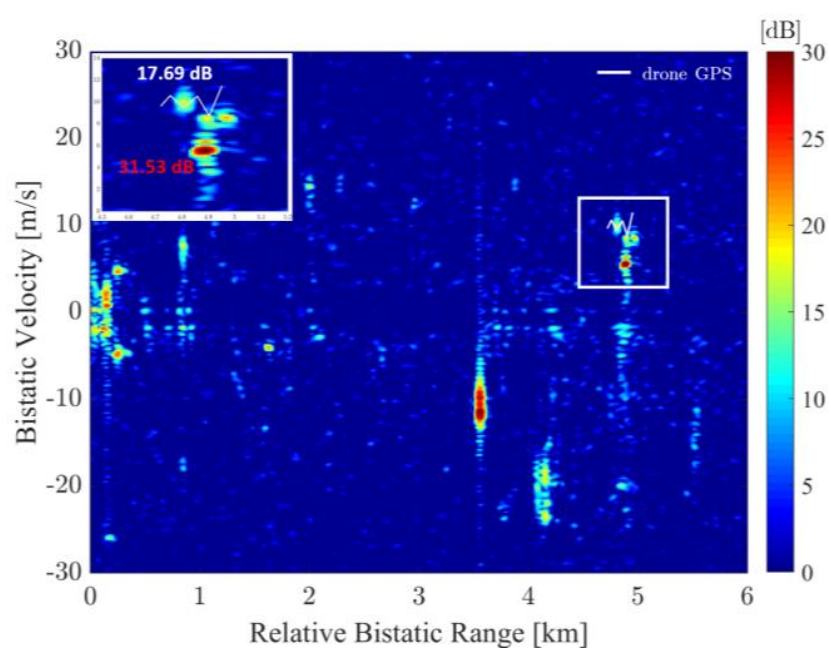

Fig. 6 Bistatic range - velocity map $\chi^{(0)}[r, v]$ obtained with $\mathrm{T}=1 \mathrm{~s}$

\section{B. Results}

The signal processing steps of Fig. 2 and detailed in Section II are applied against the available dataset. In detail, first we resort to the sliding version of the ECA (ECA-S) for the disturbance removal [11]. Then the CAF is evaluated by adopting a CPI of $\mathrm{T}=1 \mathrm{~s}$.

As a first example of the results obtained against the real data, in Fig. 6 we report the bistatic range - velocity map obtained at a single data file among the consider dataset. The map has been normalized to the thermal noise in order to measure the target SNRs. The white line represents the available GPS data for the cooperative drone at the considered time interval. Moreover, an enlarged view around the drone position is reported in the upper left corner of the figure.

By observing Fig. 6, a peak with $\mathrm{SNR}=17.69 \mathrm{~dB}$ is visible around the bistatic location $[4.81 \mathrm{~km}, 10 \mathrm{~m} / \mathrm{s}]$ and is likely to be the return from the drone. Simultaneously, other peaks are visible in the enlarged view figure, among which the highest one at position $[4.88 \mathrm{~km}, 5.6 \mathrm{~m} / \mathrm{s}]$ is received with a SNR = $31.53 \mathrm{~dB}$. In addition, a number of other peaks are visible in the map which, as reported in [9], are likely to correspond to other targets moving on the monitored area, e.g. cars, small aircraft as well as birds. This confirms that airport terminal areas are very likely to be a high-density target scenario thus a proper strategy to handle this problem is needed.

In Fig. 7 (a-b) we report the results obtained by applying the entire processing scheme shown in Fig. 2. Specifically, in Fig. 7(a) we compare the Doppler cut, before and after one iteration of the proposed strategy, obtained in the range bin where the strongest peak around the drone was present, namely at 4.88 $\mathrm{km}$. We notice that a wide notch is obtained after the proposed algorithm (see dashed red curve) and the strongest target is removed. This is also confirmed by observing Fig. 7(b), where we report the cleaned version of the range-velocity map after it also underwent a DMC stage.

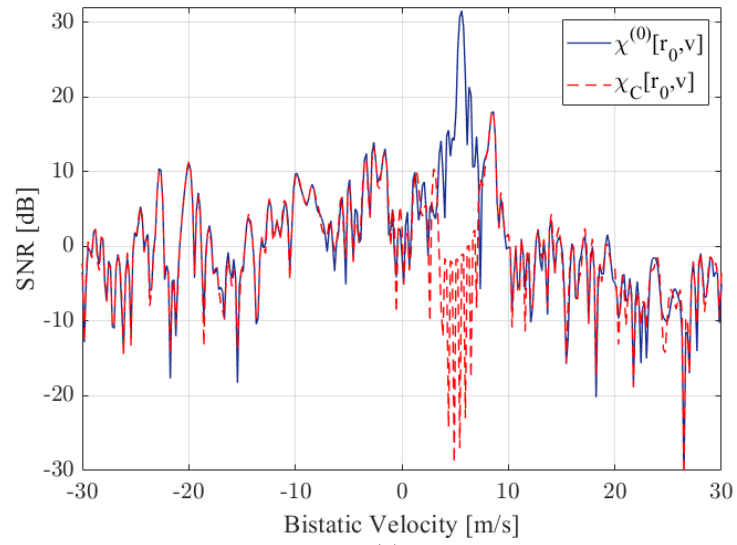

(a)

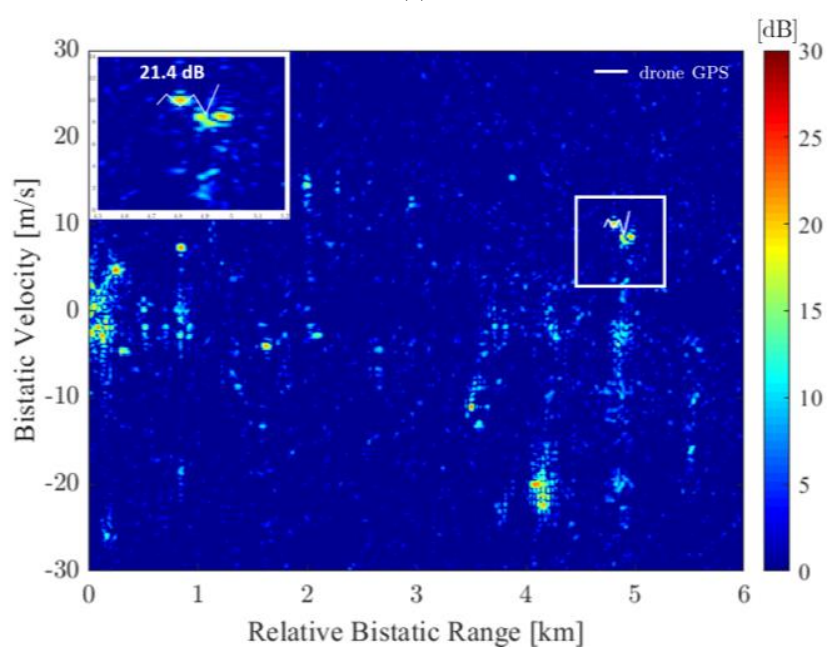

(b)

Fig. 7 Obtained results after the proposed strategy: (a) Comparison between $\chi^{(0)}\left[r_{0}, v\right]$ and $\chi_{C}\left[r_{0}, v\right]$ after the first algorithm iteration

(b) $\chi_{C}[r, v]$ after DMC

By observing the Fig. 7(b) and the enlarged view around the drone:

- the drone peak is now well visible and can be easily distinguished from the background. This improvement is also due to the proper compensation of the target motion.

- The strongest target is removed from the map thanks to the proposed algorithm, thus enabling the detection of another weak peak that was covered by its sidelobes.

- Finally, by comparing Fig. 7(b) and Fig. 6, many other strong peaks are removed from the map. It is likely to allow weaker peaks to appear.

\section{CONCLUSION}

In this work, we addressed the detection of small drones and UAVs in high-density target scenarios where the reflections from small targets might be masked by high-RCS objects.

We handled this issue by using long CPIs and by implementing a CLEAN-like multistage algorithm to remove the strongest target contributions. First, we demonstrated the

(C) 2020 IEEE. Personal use of this material is permitted. Permission from IEEE must be obtained for all other uses, in any current or future media, including reprinting/republishing

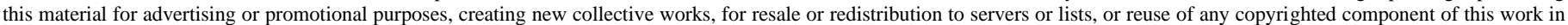
other works. 
effectiveness of the proposed strategy against simulated data representing a typical scenario. Then, a preliminary experimental result is shown, obtained against data collected by the DVB-T based AULOS ${ }^{\circledR}$ passive radar developed by Leonardo S.p.A.. The reported results show the effectiveness of the proposed strategy to tackle the problem under consideration and demonstrate the capability of DVB-T based passive radar for airport terminal area surveillance.

\section{ACKNOWLEDGEMENTS}

The authors would like to thank Dr. Roberta Cardinali from Leonardo S.p.A. for setting the experimental setup and for her technical support.

\section{REFERENCES}

[1] M. Ritchie, F. Fioranelli, H. Borrion, "Micro UAV crime prevention: Can we help Princess Leia?, " in Preventing Crime in the 21st Century, B. L. Savona and Ed. Cham, Swizerland: Springer, 2017, pp. 359-376.

[2] X. Shi, C.Yang, W. Xie, et al. "Anti-Drone System with Multiple Surveillance Technologies: Architecture, Implementation, and Challenges," IEEE Communications Magazine, 2018, 56, (4), pp. 68-74.

[3] https://www.newscientist.com/article/2190096-drones-arecausingairport-chaos-why-cant-we-stop-them/.

[4] Special Issue on Passive Radar Systems (Guest Editor: Howland P. E.), IEE Proceedings on Radar, Sonar and Navigation, 2005, 153 (3), pp. 105223.

[5] Y Liu, X.Wan, H. Tang, et al. "Digital television based passive bistatic radar system for drone detection," IEEE Radar Conference 2017, Seattle, WA, May 2017, pp. 1493-1497.

[6] D. Poullin, "Countering illegal UAV flights: passive DVB radar potentiality", 19th International Radar Symposium (IRS), Bonn, June 2018, pp. 1-10.

[7] N. del-Rey-Maestre, et al. "Optimum Beamformin to Improve UAV's detectoon Using DVB-T Passive Radars," IEEE International Radar Conference 2019, Toulon, France, Sept. 2019.

[8] T. Martelli, F. Colone, R. Cardinali, "Simultaneous short and long range surveillance of drones and aircrafts with DVB-T based Passive Radar, " IEEE International Radar Conference 2019, Toulon, France, Sept. 2019

[9] T. Martelli, F. Colone and R. Cardinali , "DVB-T based Passive Radar for simultaneous counter drone operations and civil air traffic surveillance," IET Radar, Sonar \& Navigation, in print.

[10] F. Filippini, T. Martelli, F. Colone and R. Cardinali, "Exploiting long coherent integration times in DVB-T based passive radar systems," 2019 IEEE Radar Conference (RadarConf), Boston, MA, USA, 2019, pp. 1-6.

[11] F. Colone, C. Palmarini, T. Martelli et al.: "Sliding extensive cancellation algorithm for disturbance removal in passive radar", IEEE Transactions on Aerospace and Electronic Systems, 2016, 52, (3), pp. 1309-1326.

[12] T. Jenho and B. D. Steiberg, "Reduction of sidelobe and speckle artifacts in microwave imaging: the CLEAN technique," IEEE Transactions on Antennas and Propagation, vol. 36, no. 4, pp. 543-556, Apr. 1988. 